\title{
MAPAS MENTAIS E FLASHCARDS NO ENSINO DE ESPANHOL
}

\author{
MIND MAPS AND FLASHCARDS FOR TEACHING SPANISH
}

\author{
Augusto Weiand ${ }^{1}$, Patrícia da Silva Campelo Costa Barcellos ${ }^{2}$
}

\begin{abstract}
RESUMO: Este trabalho demonstra a utilização de flashcards e mapas mentais, apoiada pela Teoria Conectivista e pela Teoria da Atividade para o ensino de espanhol como língua estrangeira. Através desses recursos tecnológicos e teorias educacionais, desenvolveu-se uma pesquisa aplicada com duas turmas do ensino fundamental de espanhol, em uma escola pública no estado do Rio Grande do Sul, da qual participaram 50 estudantes. Através de pesquisas (WAZIR et al., 2018; RAHMA, 2016; NAIR; FAREI, 2017), observa-se a eficácia da utilização de flashcards e mapas mentais de maneira separada. Contudo, o presente estudo tratou de unificar essas propostas de maneira inovadora, a qual resultou em um aumento médio de quase um ponto nas notas finais dos alunos da disciplina, se comparados o ano da aplicação do estudo com o anterior. Da mesma forma, qualitativamente foram observados ganhos tanto na aquisição da língua estrangeira, quanto no engajamento dos aprendizes e professores envolvidos.
\end{abstract}

PALAVRAS-CHAVE: mapas mentais; flashcards; aprendizado de espanhol.

ABSTRACT: This work demonstrates the use of flashcards and mind maps, supported by Connectivist and Activity Theory for the teaching of Spanish as a foreign language. Through these technological resources and educational theories, an applied research was developed with two classes of elementary students of Spanish in a public school in the state of Rio Grande do Sul, where 50 students participated. Through researches (WAZIR et al., 2018; RAHMA, 2016; NAIR; FAREI, 2017), the effectiveness of using flashcards and mind maps is observed separately. However, the present study tried to unify them in an innovative proposal, which resulted in an average increase of almost one point in the final grades of students of the discipline, comparing the year of the study to the previous one. Likewise, qualitatively gains were observed both in the acquisition of the foreign language and in the engagement of those learners and teachers involved.

KEYWORDS: mind maps; flashcards; learning of Spanish.

\section{Introdução}

A utilização de flashcards e mapas mentais no auxílio do aprendizado em sala de aula

\footnotetext{
${ }^{1}$ Doutorando do Programa de Pós-Graduação em Informática na Educação da Universidade Federal do Rio Grande do Sul (PPGIE/UFRGS).

${ }^{2}$ Professora do Programa de Pós-Graduação em Informática na Educação da Universidade Federal do Rio Grande do Sul (PPGIE/UFRGS).
} 
vem sendo demonstrada por diversos autores através dos anos (KRAUSE et al., 2014; WAZIR et al., 2018; SHAKOURI; MEHRGAN, 2012; RAHMA, 2016; KOMACHALI et al., 2012; NAIR; AREI, 2017; WEIAND et al., 2019) como um aliado ao processo de internalização do conhecimento, e consequente auxiliar para os estudos.

Um flashcard tradicional consiste basicamente em um papel pautado onde são anotados pontos importantes para a memorização de conteúdos. Dentre as diversas formas de utilizá-lo, uma delas consiste em um questionamento em uma das faces do papel e, no verso, a resposta. Além disso, alguns indivíduos escrevem termos-chave da matéria em uma face e a explicação sobre eles na outra (WAZIR et al., 2018; RAHMA, 2016). Deste modo, após o tempo dedicado às aulas, os flashcards são utilizados para auxiliar na compreensão do conteúdo estudado.

Os mapas mentais, por sua vez, foram desenvolvidos por Tony Buzan no final da década de 1960 e são definidos frequentemente através da frase "uma imagem vale mais que mil palavras". Possivelmente esta colocação decorre em virtude dos seus conceitos, organização e visualização. Com o uso de ilustrações constrói-se um mapa relacionando as informações sobre o tema, conectando-as de acordo com suas afinidades, criando uma rede. Buzan afirma que a utilização da técnica possibilita uma melhor integração entre os hemisférios esquerdo e direito do cérebro humano, tendo em vista que o esquerdo tende a ser mais verbal e analista, ao ponto que o direito é visual e perceptivo, procurando ser "uma ferramenta amigável ao cérebro", organizando e tornando o aprendizado mais flexível (BARROS; SILVEIRA, 2013; NAIR; FAREI, 2017; BUZAN, 2019; BUZAN; BUZAN, 1993).

A ilustração de um mapa mental se assemelha a uma rede, onde cada nó descreve um conhecimento, que se conecta com outro nó - o qual provavelmente possua semelhança com ele -, formando, então, uma rede de conhecimentos interligados que vão ao encontro da teoria Conectivista.

Neste contexto, sob o ponto de vista educacional, o Conectivismo, descrito por Siemens (2006, 2014), constitui-se como uma teoria de aprendizagem alicerçada nas mudanças vividas na sociedade digital, onde o processo de aprendizagem não está mais focado de forma individualista, mas nas redes estabelecidas.

Siemens (2006) descreve-a como integrada aos princípios explorados pelo Caos, Rede 
e Teoria da Complexidade e Auto-organização ${ }^{3}$, os quais são responsáveis pela aprendizagem no Conectivismo. O autor ressalta que muitos ambientes de aprendizagem demonstram-se locais onde o processo de aprender torna-se nebuloso ante esses princípios, os quais, devido às atualizações regulares, estão em constante mudança e também não integralmente sob o controle dos indivíduos (WITT; ROSTIROLA, 2020; SIEMENS, 2006).

A utilização de teorias de aprendizagem antropológicas contemporâneas demonstra que a aprendizagem ocorre apenas no contexto de atividade significativa, desta forma, torna-se importante analisar a atividade e o contexto como parte do processo de design instrucional. É inclusive necessária a análise do contexto relevante para o indivíduo, assim como da atividade (JONASSEN; ROHRER-MURPHY, 1999).

Neste sentido, a Teoria da Atividade (TA) fundamenta-se na psicologia históricocultural de Vigotsky, que descreve a noção de que a relação do Homem com o mundo não é direta, mas mediada. Baseando-se nisso, Leontiev (2009) descreve a atividade como dirigida a um objeto, o qual serve para diferenciar uma atividade da outra, pois esse é quem fornece um motivo para ela ocorrer (ARAÚJO et al., 2017).

Sob a luz dessas abordagens tecnológicas e educacionais referentes ao Conectivismo e à Teoria da Atividade, o presente trabalho apresenta o desenvolvimento de uma pesquisa que busca a resposta para a seguinte questão: "mapas mentais e flashcards podem auxiliar no aprendizado de espanhol como língua estrangeira?". Para isto, foi efetuado um experimento em uma escola da rede estadual de ensino no Rio Grande do Sul, em uma disciplina de Espanhol, o qual envolveu 46 alunos de duas turmas do sétimo ano do ensino fundamental. O objetivo dessa prática consistiu em analisar se a utilização das abordagens descritas na pergunta de pesquisa auxiliou no aprendizado de espanhol como língua estrangeira.

Contudo, como descrito no estudo de Weiand et al. (2019), inexiste ainda uma abordagem única que contemple flashcards e mapas mentais utilizados conjuntamente no ensino/estudo de forma presencial ou virtual. Desta maneira, este trabalho desenvolveu primeiramente a proposta de forma offline, ou seja, os artefatos utilizados - mapas mentais e flashcards - foram apenas confeccionados com auxílio de software e, posteriormente,

\footnotetext{
${ }^{3}$ Conforme Teixeira e Rostirola (2020), a teoria do Caos se integra ao Conectivismo em vista "da forma imprevisível como o conhecimento é organizado na sociedade, arranjos complexos a qual cada 'Nó' encontra um significado" (p. 2). Os autores descrevem ainda que o conhecimento sempre teve o caráter de Rede, servindo de base para a aprendizagem humana desde antes da tecnologia. Os conceitos de nós e Redes de Conhecimento advém da teoria da Complexidade de Edgar Morin (2011), os quais retratam o funcionamento da sociedade complexa - a sociedade em que o conhecimento é um tecido formado por interconexões. Já a Auto-organização é estabelecida através das relações entre os nós da rede, os quais são de difícil previsibilidade, além da capacidade de criação de padrões comportamentais imprevisíveis.
} 
impressos para uso em papel. A troca e a manipulação desses artefatos foram realizadas de forma física durante o desenvolvimento desta pesquisa em virtude das condições tecnológicas disponibilizadas pela escola participante do estudo.

De forma a contemplar o objetivo proposto neste estudo, este artigo está organizado da seguinte forma: na seção 1, são descritos os autores que fundamentaram a presente proposta; já na seção 2, é descrita a metodologia utilizada para o desenvolvimento e aplicação da pesquisa; em 3, são descritos os resultados obtidos; por fim, na seção 4, são descritas as conclusões efetuadas, tendo como base os trabalhos revisados e a pesquisa aplicada.

\section{Referencial Teórico}

Assim como as formas de obter informações estão em constante alteração, a maneira como os indivíduos aprendem acompanha essas mudanças. $\mathrm{O}$ aprendizado em rede é uma realidade presente no cotidiano de todos, e o conhecimento está em constante atualização, permitindo-nos utilizar as conexões de pessoas - ou equipamentos - para buscá-lo ou atualizálo de maneira eficaz. Assim como novas tecnologias são desenvolvidas, teorias de aprendizagem baseadas nesses novos aprendizados também são. Sob este viés, esta seção busca delinear as abordagens metodológicas e teorias envolvidas neste estudo.

\subsection{Flashcards}

Diversos autores demonstram a utilização de flashcards como fortes aliados no auxílio ao aprendizado em sala de aula (KRAUSE et al., 2014; WAZIR et al., 2018; SHAKOURI; MEHRGAN, 2012; RAHMA, 2016), podendo auxiliar especialmente na aquisição de vocabulário.

As pesquisas realizadas com o uso de flashcards na área do aprendizado de línguas são amplas, e Wazir et al. (2018), por exemplo, descrevem um estudo teórico sobre a sua eficácia no aprendizado de vocabulário do inglês como segunda língua s. O número de estudantes que têm um amplo vocabulário é baixo, e o uso de flashcards pode implicar diretamente no seu aprendizado (Wazir et al., 2018).

Já Rahma (2016) desenvolveu sua tese de doutorado utilizando flashcards para aprimorar o vocabulário de estudantes do oitavo ano da MTsN Godean2, nos anos de 2016 e 2017. Durante o estudo foram efetuadas entrevistas e aplicação de pré e pós-teste, de modo a 
possibilitar análises qualitativas e quantitativas, estas últimas resultando em um aumento médio de 100\% nas notas dos estudantes envolvidos (Rahma, 2016).

A utilização de flashcards para o ensino de vocabulário de língua estrangeira foi o tema de pesquisa de Komachali et al. (2012), na qual os autores desenvolveram um estudo com 50 participantes pré-universitárias de uma escola pública voltada para meninas no Irã. Os resultados demonstraram melhora substancial no aprendizado, o que, segundo as próprias estudantes, se deve ao fato de haver uma maior significação do conteúdo para elas. Em virtude de ser uma estratégia simples e divertida, as aprendizes puderam desenvolver os flashcards com imagens e outros recursos visuais (Komachali et al., 2012).

\subsection{Mapas Mentais}

Os mapas mentais desenvolvidos por Tony Buzan (2019) baseiam-se no conceito de pensamento radiante, que é definido analogamente às ramificações de uma árvore, ou mesmo à rede complexa do sistema nervoso humano, e proporciona a utilização dos dois hemisférios cerebrais: o esquerdo (lógico) e o direito (criativo). A figura 1 ilustra um exemplo de mapa mental.

Figura 1. Exemplo de Mapa Mental

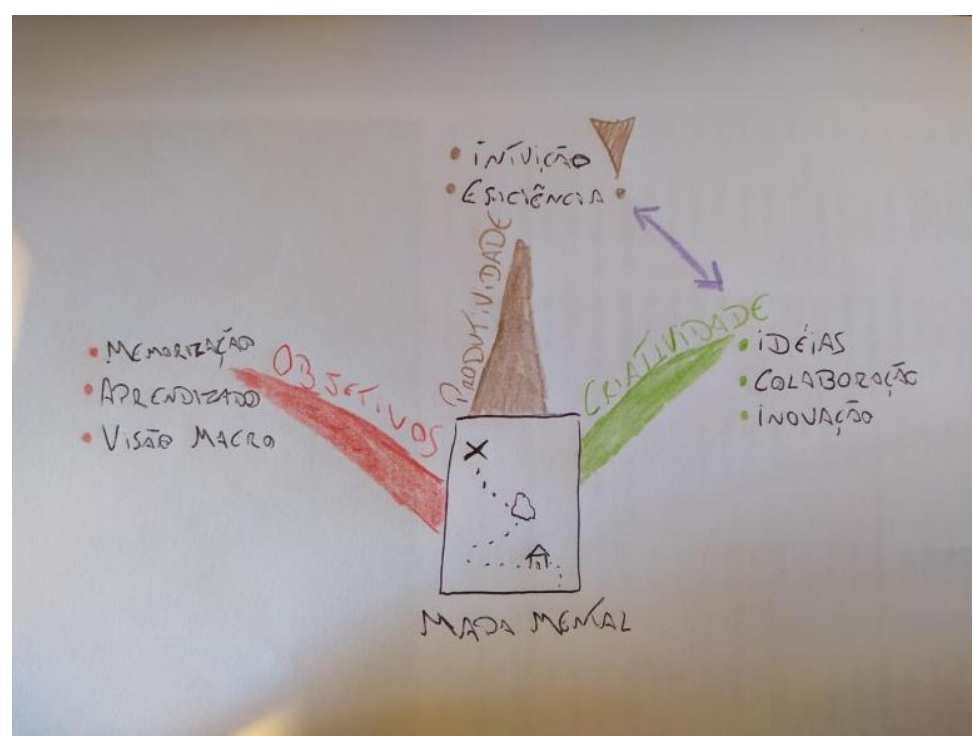

Fonte: Autores

Autores como Zarzo (2015) e Nair e Farei (2017) descrevem pesquisas que foram 
realizadas com o uso de mapas mentais, de modo a possibilitar a melhora na memorização e consequente aprendizagem. Zarzo (2015) utilizou um software de mapas mentais de forma integrada ao AVA Moodle, com o propósito de desenvolver uma abordagem de organização metacognitiva do conhecimento. Para isto, foram efetuadas diversas atividades educacionais seguindo as regras clássicas da mnemônica para alunos do ensino médio, na disciplina de filosofia. Os autores descrevem que ficou evidenciada a memória figurativa associada ao mapa mental, e essa contribuiu favoravelmente no desenvolvimento das habilidades analíticas e sintéticas, assim como da capacidade crítica. Ainda, apontam que a atividade de elaboração conjunta de glossários e mapas mentais reforçou a capacidade de trabalhar de forma colaborativa, contribuindo para a conscientização de um senso de responsabilidade individual no processo de ensino e aprendizagem (Zarzo, 2015).

Nair e Farei (2017), por sua vez, utilizaram mapas mentais como ferramenta para que os alunos de Graduação e Pós-Graduação da área de informática concluíssem com êxito tarefas de avaliação baseadas em pesquisas. A motivação dos autores é baseada no fato de que, como descrito anteriormente, os mapas mentais buscam se assemelhar às estruturas do cérebro humano, facilitando a compreensão e, consequentemente, o aprendizado. Além disso, os autores citam outros benefícios, tais como: memória e recordação; facilitação do processo de aprendizagem; apresentações de ideias mais claras; promoção de colaboração em grupo; organização e associação de pensamentos; melhora na habilidade de escrita; fomento à criatividade e ao pensamento crítico; resolução de problemas; debates; entre outros. Os estudos evidenciaram que a utilização dos mapas mentais auxiliou os alunos na compreensão do processo de pesquisa e na sua aplicação.

\subsection{O Conectivismo e a Teoria da Atividade}

O Conectivismo, segundo a abordagem descrita por Siemens (2006, 2014), constitui-se em uma teoria de aprendizagem alicerçada nas mudanças vividas na sociedade digital, onde o processo de aprendizagem não está mais focado de forma individualista, mas nas Redes estabelecidas.

Contudo, o conceito central desta teoria, as Redes, surge antes da era digital:

As Redes têm servido de base para a aprendizagem humana bem antes da tecnologia que se vê na sociedade atual. O desenvolvimento de competências na caça, coleta e agricultura exigiam conhecimentos a serem compartilhados a cada nova geração: na 
atividade agrícola a geração mais jovem foi construída sobre o trabalho das outras. Pequenos avanços e novas técnicas e ferramentas serviram para melhorar continuamente disciplinas, como agricultura, ferraria, soldadura e, mais recentemente, a filosofia e as ciências (Siemens, 2006, p. 1).

A era digital veio auxiliar nesse conceito, a comunicação e o compartilhamento se tornaram simples e ágeis, assim como possíveis de serem feitos em qualquer local e horário. Tais mudanças recorrentes do conhecimento alicerçam a Teoria do Conectivismo (GONZALEZ, 2004).

Considera-se um mundo onde muitas pessoas - as quais talvez não possuam nenhuma relação entre si - provavelmente atuarão em diversas áreas. A aprendizagem informal passa, então, a ser tratada de forma semelhante à formal, ocorrendo de diversas maneiras comunidades de prática $^{4}$, redes sociais, tarefas de trabalho, etc. A aprendizagem dura a vida

inteira, e o indivíduo não está completamente formado após completar a educação formal. O saber como e o saber o quê está sendo complementado com o saber onde - na sua "Rede" de contatos/conhecimentos (SIEMENS, 2006).

Percebe-se, assim, que a Teoria Conectivista permite a utilização de conceitos atuais para sua definição, principalmente com relação às conexões como atividades de aprendizagem. Assim, Stephenson (2005) descreve uma posição relevante sobre isso:

\footnotetext{
A experiência tem sido considerada a melhor mestra do conhecimento. Como não podemos experimentar tudo, as experiências de outras pessoas e, consequentemente, as outras pessoas, tornam-se substitutas do conhecimento. 'Eu guardo meu conhecimento nos meus amigos' é um axioma para coletar conhecimento através da coleta de pessoas (Stephenson, 2005).
}

Através desse trecho, considera-se que a aprendizagem que reside fora de nós, seja dentro de uma organização ou em um banco de dados, pode nos auxiliar, através da interconexão das informações especializadas nesses locais (Siemens, 2006). Essas informações acessadas pelas pessoas que participam das atividades relacionadas ao aprendizado em questão introduzem a Teoria da Atividade (TA), a qual, assim como o Conectivismo, considera que o conhecimento envolve todo o sistema (equipes, organizações, ambiente, histórico), além de apenas um autor ou usuário.

A TA surge a partir dos trabalhos de Leontiev (1983), contudo muitos autores

\footnotetext{
${ }^{4}$ As comunidades de prática se constituem de pessoas que compartilham um interesse comum sobre um determinado assunto, tendo ou não domínio sobre ele (PIVETTA, 2016).
} 
acabaram por adotar essa denominação também para se referirem aos trabalhos de Vigotsky, Luria e outros integrantes dessa escola da Psicologia (DUARTE, 2003). A TA não é uma metodologia, mas uma "estrutura filosófica para estudar diferentes formas de práxis humana como processos de desenvolvimento, ambos os níveis individual e social interligados ao mesmo tempo" (JONASSEN; ROHRER-MURPHY, 1999).

Essa teoria acredita que a mente humana emerge e existe como um componente de interações com o meio, então a atividade é precursora do aprendizado, se materializando através das interações humanas intencionais e conscientes com o mundo objetivo e as atividades que fazem parte dessas interações. Seu foco é a interação sujeito-objeto (ARAÚJO et al., 2017). Com isso, ela se constitui em um modo eficaz de análise sócio-cultural e sóciohistórico, através do qual podem-se analisar a maioria das formas de atividade humana. Como praticamente tudo na vida, a análise da atividade não pode ser realizada fora de seu contexto, correndo o risco de interpretações equivocadas, perpassando, então, pelos tipos de atividades, os objetivos de todos, quem está envolvido, objetos ou produtos resultantes da atividade, suas intenções e regras (JONASSEN; ROHRER-MURPHY, 1999).

Aprender e fazer são inseparáveis e são iniciados por uma intenção, a qual é direcionada ao(s) objeto(s) da atividade, que pode(m) ser qualquer coisa, como um programa de computador, objetos conceituais como uma tese, códigos de programação, uma conversa de trabalho entre docentes, entre outros.

Este processo de transformação do objeto, como por exemplo o desenvolvimento desta pesquisa, move o sujeito à busca de seu objetivo, continuando a motivar a atividade, por exemplo, finalizar a escrita. Desta forma, o objeto da atividade - desenvolvimento desta pesquisa -, concentra as ações pretendidas nele próprio, sendo também o motivo da atividade.

Durante esse processo, constantemente são utilizados artefatos como: signos, procedimentos, máquinas, métodos, leis e formas de organização de trabalho. Essa teoria descreve esse fato como uma constante, em virtude de que esses artefatos ou ferramentas medeiam ou proporcionam a alteração da natureza da atividade humana, assim como quando internalizados influenciam o desenvolvimento mental (JONASSEN; ROHRER-MURPHY, 1999).

Um processo importante que deve ser analisado é quanto à estrutura da atividade (todas as atividades que envolvem o assunto), que define o objetivo do sistema de 
atividades. As atividades consistem em ações individuais e cooperativas e cadeias de operações. Essa hierarquia de atividades, ações e operações descreve a estrutura de atividades (JONASSEN; ROHRER-MURPHY, 1999, p. 13).

Jonassen e Rohrer-Murphy (1999) descrevem três níveis de estrutura da Teoria da Atividade. O nível de atividade (LINARD, 1995) é interpretado como nível intencional, pois foca nas intenções ou motivações, descrevendo uma perspectiva do objetivo, como por exemplo o planejamento desta pesquisa. Já o nível de ação é o nível funcional (LINARD, 1995), que utiliza ações de planejamento e resolução de problemas para cumprir as atividades. Continuando no mesmo exemplo, o nível funcional seriam as atividades de desenvolvimento dos artefatos para a aplicação do estudo, a escrita deste artigo, etc. Por fím, têm-se as operações, as quais são descritas como comportamentos automatizados ou rotinizados, tais como tabulação e desenho de gráficos dos dados relacionados à aplicação deste estudo.

Em conjunto, esses três níveis (atividade, ação e operação) compreendem a estrutura de uma atividade, demonstrando suas inter-relações entre o pensamento consciente e inconsciente e a performance focada nos objetos (JONASSEN; ROHRER-MURPHY, 1999).

\subsection{Considerações}

Com o apoio dos autores descritos nas seções anteriores, pode-se observar que existem diversas pesquisas relacionando as abordagens propostas para o presente estudo de maneira distinta. Os flashcards são uma importante fonte de auxílio para aprendizagem, com diversas formas de utilização descritas na literatura estudada. Neste mesmo contexto, os mapas mentais descrevem metodologias de organização, proporcionando melhor significação do conteúdo abordado.

Existem, então, evidências que demonstram a utilização dessas abordagens de forma vantajosa para o aprendizado de línguas. Contudo, também demonstrou-se evidente a inexistência de abordagens que demonstrem a sua eficácia combinada, ou seja, a utilização de flashcards e mapas mentais conjuntamente, de forma a contribuir no aprendizado de línguas.

Sob esta perspectiva, a Teoria Conectivista (SIEMENS, 2006, 2014), que se baseia no conceito de Redes para o processo epistemológico e de aprendizagem - análoga aos mapas mentais - , aliada à Teoria da Atividade (LEONTIEV, 1978), a qual estabelece os elementos fundamentais no processo de aprendizagem mediada/social, auxilia no alicerce da proposta desenvolvida neste trabalho. 
Através deste diálogo e bases teóricas, foi desenvolvida uma pesquisa aplicada que será descrita a seguir.

\section{Metodologia}

A proposta metodológica desta pesquisa é de natureza mista (qualitativa e quantitativa), de cunho interpretativista, tendo em vista as ações planejadas de observação das atividades propostas para desenvolvimento da língua estrangeira. Esta escolha está baseada em afirmações de diversos autores como Pinheiro (2011), Rosa (2018) e Esteban (2010), que descrevem a pesquisa de natureza mista como adequada para a investigação do pesquisador, tendo em vista sua interpretação no processo, possibilitando ângulos de análise e reflexões diversas. Através desses elementos, estuda suas subjetividades, que não podem ser tratadas apenas quantitativamente, pois muitas vezes escondem a dimensão humana, pluralidade e interdependência dos fenômenos estudados.

A presente pesquisa foi desenvolvida em conjunto com uma professora da disciplina de Espanhol de uma escola estadual do sul do Brasil, durante 10 períodos de 50 minutos cada, totalizando 10 semanas de pesquisa. A professora regente da disciplina, em conjunto com os pesquisadores, desenvolveu um mapa mental e seis flashcards sobre o conteúdo abordado. Participaram do estudo, 46 alunos de duas turmas (descritas como Turma A e Turma B, neste trabalho), do sétimo ano do ensino Fundamental.

O mapa mental utilizado relaciona tópicos sobre a classe gramatical dos adjetivos, de forma complementar às explicações da professora. Juntamente, houve utilização dos flashcards (figuras 2 e 3), de modo que esse material foi impresso e entregue para todos os alunos. 
Figura 2. Mapa Mental entregue aos alunos

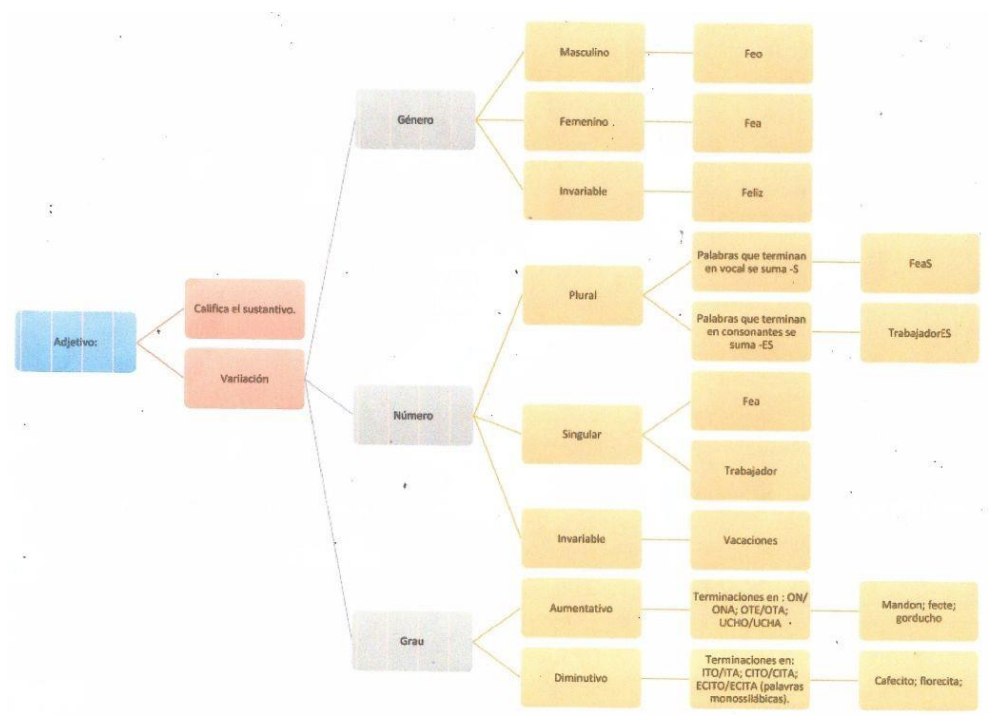

Fonte: Autores, 2020.

Figura 3. Flashcards entregues aos alunos
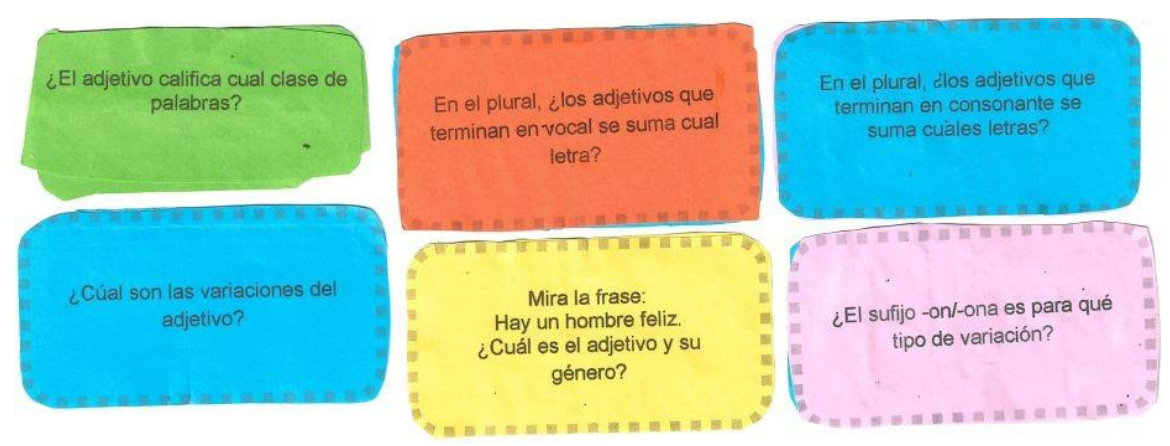

Fonte: Autores, 2020.

Para a confecção do mapa conceitual foi utilizada a ferramenta Microsoft Word, já para os flashcards, o Google Docs. A aplicação transcorreu de forma offline em virtude da escola não dispor de infraestrutura de informática operante para uso acadêmico, assim como haver ausência de sinal de internet móvel de qualidade e dispositivos móveis para o desenvolvimento das atividades. Contudo, esta aplicação é considerada experimento piloto de uma tecnologia que será desenvolvida futuramente na modalidade online, incluindo as abordagens propostas por Weiand et al. (2019) e pelo presente trabalho.

Sendo assim, o desenvolvimento da pesquisa foi organizado e desenvolvido em quatro momentos:

- No primeiro momento os alunos foram apresentados ao conteúdo de forma 
tradicional: conhecimento teórico e explicação expositiva. Como atividade prática fizeram exercícios de fixação;

- Posteriormente, cada aluno recebeu um mapa mental (figura 2) e seis flashcards

(figura 3), os quais serviram de material de revisão e estudos individuais;

- A próxima etapa possibilitou ao aluno um papel mais ativo no seu aprendizado, solicitando que ele desenvolvesse ao menos seis flashcards para complementar seus estudos eque esses pudessem ser compartilhados entre eles;

- Por fim, foi disponibilizado um questionário para cada estudante com perguntas relacionadas sobre a prática efetuada e como ela auxiliou (ou não) o entendimento do conteúdo.

Ao final das 4 semanas de aula, a professora aplicou sua avaliação sobre o tópico estudado e, na sequência, um questionário que dispunha de cinco questões baseadas na escala Likert (LIKERT, 1932) e uma descritiva, detalhadas a seguir.

Q1. A utilização de mapas mentais auxiliou o aprendizado?

Q2. A utilização de flashcards auxiliou o aprendizado?

Q3. A possibilidade de manipulação do mapa mental, sugerindo e/ou editando-o a sua vontade, auxiliou no entendimento do conteúdo?

Q4. A troca de flashcards entre os colegas auxiliou no entendimento do conteúdo?

Q5. A comunicação com colegas e professora, relacionada a cada tópico do mapa mental, foi importante para o entendimento do conteúdo?

Q6. Você considera que os flashcards e mapas mentais foram relevantes para o seu aprendizado? Por quê?

Essas questões foram aplicadas de modo a possibilitar análises qualitativas e quantitativas da aplicação ao final da pesquisa, detalhadas a seguir.

\section{Resultados}

Do ponto de vista quantitativo, comparando as notas finais dos alunos do ano de 2018 com os de 2019 (ano de aplicação do estudo), foram elaboradas as tabelas 1, 2 e 3, que relacionam o ano, a turma (com seu pseudônimo) e as notas dos alunos. 


\begin{tabular}{|c|c|c|c|c|}
\hline Ano & Número de Alunos & Nota Mínima & Nota Máxima & Nota Média \\
\hline 2018 & 37 & 1 & 6,9 & 4,4 \\
\hline 2019 & 46 & 1,5 & 7,5 & 5,3 \\
\hline
\end{tabular}

Tabela 1. Relações entre ano, número de alunos e notas

\begin{tabular}{|c|c|c|c|c|}
\hline Turma & Número de Alunos & Nota Mínima & Nota Máxima & Nota Média \\
\hline A & 22 & 1,5 & 6,9 & 4,1 \\
\hline B & 15 & 1 & 6,8 & 4,8 \\
\hline
\end{tabular}

Tabela 2. Relações entre as turmas de 2018, quanto ao número de alunos e notas

\begin{tabular}{|c|c|c|c|c|}
\hline Turma & Número de Alunos & Nota Mínima & Nota Máxima & Nota Média \\
\hline A & 24 & 1,5 & 7 & 5 \\
\hline B & 22 & 3 & 7,5 & 5,6 \\
\hline
\end{tabular}

Tabela 3. Relações entre as turmas de 2019, quanto ao número de alunos e notas

Observa-se que no ano de 2018 as turmas foram ligeiramente menores. Contudo, este fator não se monstrou relevante para a estatística que apresentou aumento em todas as notas, tanto nas mínimas, quanto nas máximas. Destaca-se também o aumento considerável das médias em ambas as turmas, as quais ficaram em 0,9 e 0,8 respectivamente.

Figura 4. Respostas do questionário

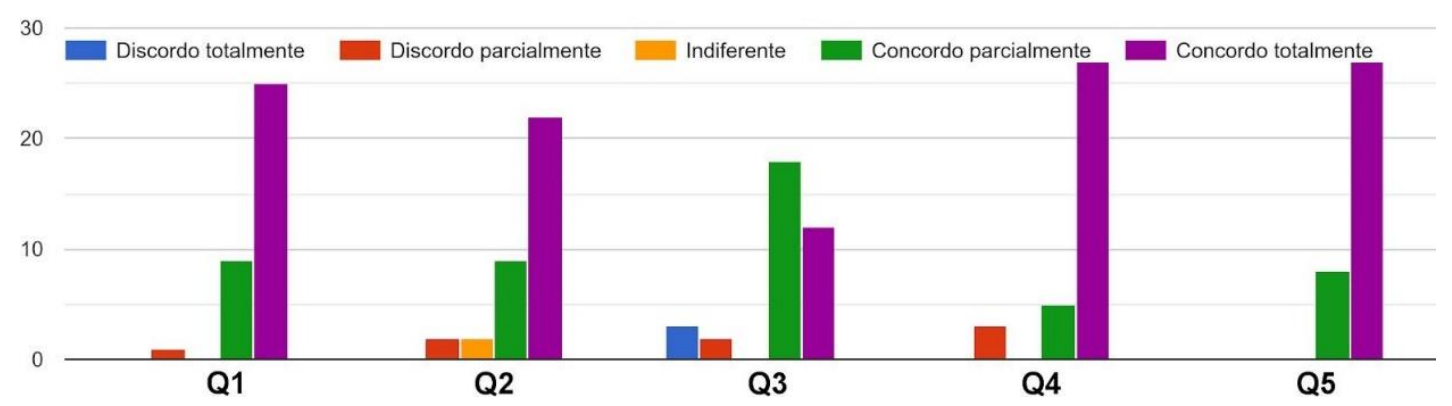

Fonte: Autores, 2020

Somam-se a esses números os 35 participantes que responderam aos questionários finais, os quais são demonstrados no gráfico 4.

Analisando este gráfico, percebe-se que as abordagens de mapa mental e flashcards 
foram satisfatórias em praticamente todos os quesitos estudados, apenas com exceção do questionamento Q3 - "A possibilidade de manipulação do mapa mental, sugerindo e/ou editando-o a sua vontade, auxiliou no entendimento do conteúdo?", o qual demonstrou padrão diferente de respostas.

Estas respostas podem sugerir que a manipulação não foi satisfatória, tendo em vista o esperado pelos alunos, ou quanto à própria abordagem - em papel e não digital -, que pode ter dificultado a manipulação dos mapas e flashcards.

Sob outra perspectiva, observando as questões Q4 e Q5, percebe-se que a possibilidade de troca e diálogo na rede de colaboração pode auxiliar o processo de aprendizado das atividades propostas, demonstrando que as conexões entre os indivíduos envolvidos são relevantes para o desenvolvimento destas.

Contudo, de acordo com a professora da turma que acompanhou todo o processo de desenvolvimento e pesquisa, ambas as turmas utilizaram os flashcards com motivação maior do que com os exercícios habituais de fixação. De toda maneira, foram notadas peculiaridades inerentes a cada uma.

A turma A apresentou rendimento razoável, mas com alguma dificuldade na identificação das variações - o que se percebe também observando as notas da tabela 3. Já a turma B obteve rendimento consideravelmente diferente, onde apenas um terço da turma apresentou dificuldades e obteve notas abaixo da média - mesmo assim superiores às da turma A. Essa mesma turma B demonstrou um engajamento maior no desenvolvimento das atividades, notadamente na confecção dos cartões, quanto à grafia, conteúdo e uso, visto que a própria turma elaborou um desafio para determinar quem teria mais acertos sem a intervenção da professora.

Para finalizar as atividades, individualmente cada aluno fez uma avaliação escrita onde deveria reconhecer os adjetivos utilizados em um texto, identificar as variações de gênero número e grau e fazer a transição de adjetivos de acordo com o solicitado. O resultado dessa avaliação evidenciou que os alunos da turma B obtiveram um rendimento superior ao constatado em anos anteriores e, inclusive, sobre a turma A - vide tabelas 1 e 3.

Quanto à pergunta Q6 - dissertativa, enumeramos algumas das respostas recebidas, consideradas relevantes ao presente estudo:

- "Sim, porque eu consegui entender melhor"; 
• "Sim, porque me ajudou muito para aprender e é mais rápido aprender com eles";

• "Sim foi melhor para a fixação do conteúdo";

- "Sim porque foi bem legal e divertido aprender com o mapa mental e flashcards";

- "Sim, pois é uma forma de aprendizado eficiente, rápido e divertido";

- $\quad$ SSim, porque quem tem mais dificuldade de aprender, fica mais fácil. E pra quem não tem, também ajudou bastante";

• "Sim, porque ajudo (sic) muito nos estudos";

Percebe-se que o rol de respostas - quantitativas e qualitativas, assim como os relatos da professora e alunos, demonstram que a união das metodologias de mapas mentais e flashcards pode ser benéfica para o aprendizado da língua estrangeira proposta neste estudo.

\section{Conclusões}

Tendo em vista a complexidade do conteúdo para os aprendizes do estudo e o tempo de duração da pesquisa, pode-se concluir que o uso do mapa mental e dos flashcards é um elemento que pode contribuir para a melhora da aprendizagem de uma língua adicional. Além disso, proporciona o uso livre da criatividade na confecção dos flashcards e interação dos alunos nas trocas e exercícios de perguntas e respostas.

Da mesma forma, o experimento possibilitou observar o vínculo que as teorias do Conectivismo e da Atividade demonstraram com a pesquisa apresentada. Os conceitos descritos por elas podem ser perceptíveis no que tange principalmente ao uso das redes de informação, às interconexões que elas possibilitam, e ao conhecimento envolvendo todo o sistema e não somente um indivíduo.

Ainda, foi possível observar uma lacuna relativa à manipulação dos elementos, algo que se torna mais complexo quando feito em papel. Sendo assim, uma das propostas de trabalho futuro desta pesquisa se relaciona a esse fator, de modo a desenvolver uma abordagem como a proposta aqui de maneira virtual, a fim de facilitar a utilização de flashcards e mapas mentais através de dispositivos digitais.

\section{Agradecimentos}

Agradecemos ao IFRS Campus Osório pela concessão de horário especial de estudante para o autor Augusto. 


\section{REFERÊNCIAS}

ARAÚJO, C. A. P.; SANTOS, J. D. P.; MEIRELES, J. C. D. (2017). Uma proposta de investigação tecnológica na Educação Básica: aliando o ensino de Matemática e a Robótica Educacional. Revista Exitus, 7(2):127.

BARROS, S. A. D.' SILVEIRA, D. S. D. (2013). Uma Revisão Sistemática Sobre Mapas Mentais Em Processos De Negócios. XXIII Congresso de Iniciação Científica, VII Congresso de Iniciação em Desenvolvimento Tecnológico e Inovação da UFPE, IV Encontro de Iniciação Científica do Ensino Médio da UFPE.

BUZAN, T. (2019). Dominando a Técnica dos Mapas Mentais, vol 1. Editora Pensamento Cultrix, São Paulo, 1 edition.

BUZAN, T.; BUZAN, B. (1993). The Mind Map Handbook. London BBC Books.

DUARTE, N. (2003). A teoria da atividade como uma abordagem para a pesquisa em educação. Perspectiva, 21(2):279-301.

ESLAHCAR KOMACHALI, M. M.; KHODAREZA, M.; ESLAHCAR KOMACHALI IN

TEFL, M. M.; KHODAREZA IN TEFL, M. (2012). The Effect of Using Vocabulary Flash Card on Iranian Pre-University Students; Vocabulary Knowledge. International Education Studies, 5(3).

ESTEBAN, M. P. S. (2010). Pesquisa qualitativa em educação, volume 1. Porto Alegre, amgh edition.

GONZALEZ, C. (2004). The Role of Blended Learning in the World of Technology. Benchmarks Online, 7(9):9-10.

JONASSEN, D. H.; ROHRER-MURPHY, L. (1999). Activity theory as a framework for designing constructivist learning environments. Educational Technology Research and Development, 47(1):61-79.

KRAUSE, S.; MAASS, S.; CHAN, C.; WATERS, C.; CARBERRY, A.; AND KORETSKY, M. (2014). Web-enabled formative feedback and learning resources for enhancing student attitude, achievement, and persistence. In 2014 IEEE Frontiers in Education Conference (FIE) Proceedings, pages 1-8. IEEE.

LIKERT, R. (1932). A Technique for the Measurement of Attitudes. In R. S. Woodworth, editor, Archives of Psychology, volume 22, pages 1-55. R. S. Woodworth, New York, NY, USA.

LINARD, M. (1995). New debates on learning support. Journal of Computer Assisted Learning.

NAIR, S. S. K. ; FAREI, K. A. (2017). A brain friendly tool to facilitate research-teaching nexus: Mind maps. In 2017 8th International Conference on Information and Communication Systems (ICICS), pages 82-85. IEEE. 
PINHEIRO, P. A. (2011). Práticas colaborativas de escrita por meio de ferramentas da internet: ressignificando a produção textual na escola. $\mathrm{PhD}$ thesis, Universidade Estadual de Campinas, Campinas.

RAHMA, M. A. (2016). Improving Grade Eight Students' Vocabulary Mastery Using Flashcards At Mtsn Godean In The Academic Year Of 2016/2017. PhD thesis, Yogyakarta.

ROSA, A. d. A. S. (2018). Práticas Letradas Na Formação Inicial De Professores De Espanhol. $8^{\circ}$ Encontro Internacional de Formação de Professores, 11(1):1-11.

SHAKOURI, N.; MEHRGAN, K. (2012). The Impact of Using Flash Cards on Promoting University Students' Knowledge of Vocabulary. Advances in English Linguistics (AEL), $1(2): 47-55$.

SIEMENS, G. (2006). Connectivism: Learning Theory or Pastime of the Self-Amused.

SIEMENS, G. (2014). Overview of connectivism.

STEPHENSON, K. (2005). Quantum Theory of Trust, volume 1. Pearson Education Ltd, financial edition.

TEIXEIRA WITT, D.; CRISTINA MARTINI ROSTIROLA, S. (2020). Conectivismo Pedagógico: novas formas de ensinar e aprender no século XXI. Revista Thema, 16(4):1012.

WAZIR, H. B.; OTHMAN, A.; YUSOF, Y. M. (2018). Vocabulary Flashcard in Learning English as Second Language for non-native Learners. In SMMTC Postgraduate Symposium 2018, page 280 .

WEIAND, A.; LUDOVICO, F. M.; BARCELLOS, P. D. S. C. C. (2019). Virtual Learning Environments in the Light of Mind Maps and Flashcards: a Systematic Literature Review. International Journal for Innovation Education and Research, 7(9):104-113.

ZARZO, E. (2015). The Art of Memory in the Digital Age. Procedia - Social and Behavioral Sciences, 178:222-226.

Recebido em 05/03.

Aceito em 13/06. 